\title{
Family Efficacy within Ethnically Diverse Families: A Qualitative Study
}

\section{TSUI-SUI A. KAO* CLEOPATRA H. CALDWELL ${ }^{\dagger}$}

Family efficacy, which refers to a family's belief in its ability to produce a desired outcome, has been shown to protect adolescents from risky health behaviors. Few studies have examined family efficacy within diverse populations, however, and understanding of how efficacy is framed and formed within the context of cultural and familial values is limited. This descriptive qualitative study examined sources of family efficacy within ethnically and socioeconomically diverse families, evaluating how such families develop and exercise family efficacy with the intent to protect adolescents from risky health behaviors (i.e., marijuana and alcohol use and early sexual activity). We collected qualitative data via two semi-structured interviews, 4-6 months apart, with 31 adolescents (ages 12-14) and their parent/s, for total of 148 one-on-one interviews. Thematic analysis identified three distinct domains of family efficacy: relational, pragmatic, and value-laden. Prior experiences and cultural background influenced the domain/s utilized by families. Significantly, families that consistently tapped into all three domains were able to effectively manage personal and family difficulties; these families also had family strategies in place to prevent adolescents from risky behaviors. Health professionals could utilize this concept of multidimensional family efficacy to promote health within culturally diverse families.

Keywords: Adolescents; Risky behavior; Health behavior

Fam Proc 56:217-233, 2017

$\mathrm{T}$ he emergent construct of family efficacy is significantly associated with adolescent health behaviors (Bandura, Caprara, Barbaranelli, Regalia, \& Scabini, 2011; Kao, Lupia, \& Clement-Stone, 2014; Profugo, Mendoza, \& Magno, 2009) and provides an opportunity to engage strengths-based efforts to inhibit risky adolescent behavior. But there is limited understanding of how family efficacy is framed and formed within families. This is particularly evident for ethnically diverse families, who have unique historical experiences and distinct cultural norms. The purpose of this descriptive qualitative study was to examine and define sources of family efficacy and to evaluate how ethnically diverse families develop or exercise family efficacy with the intent to protect adolescents from risky health behavior.

${ }^{*}$ School of Nursing, University of Michigan, Ann Arbor, MI.

'School of Public Health, University of Michigan, Ann Arbor, MI.

Correspondence concerning this article should be addressed to Tsui-Sui A. Kao, School of Nursing, University of Michigan, 400 N. Ingalls, RM 3347, Ann Arbor, MI 48109-5482. E-mail: anniekao@umich.edu.

The authors thank the participants, who generously shared their experiences. We also would like to extend our appreciation to the research assistants who have been involved in this project. We also would like to recognize editorial help from Sara Versluis, MA. This study was funded by a grant awarded to the first author from the Robert Wood Johnson Foundation Nurse Faculty Scholar Program (Grant ID \# 69345). 


\section{Strengths-Based Approaches}

Among diverse populations, cultural bias and stereotyping inhibit culturally responsive care (Sam \& Berry, 2010). Rather than focus on deficits, Walsh (2003) maintained that practitioners can avoid assumptions or biased interpretations of clients' cultural perspectives by focusing on sources of strength, such as how families foster and exert strengths within their cultural context. A body of literature (Fang \& Schinke, 2012; Hodge, 2001; Kumpfer \& Alvarado, 2003) supports the notion that strengths-based investigations and interventions provide the most efficacious medium for supporting and effecting behavior change.

Within family research, strengths-based approaches are often linked with the concept of family resilience, which is defined as familial traits that lead to successful adaptation and coping in response to a significant stressor or adversity (Walsh, 2003). Family resilience is often used when discussing and assessing a family's ability to overcome challenges and crises (Jaffee, Caspi, Moffitt, Polo-Tomás, \& Taylor, 2007). But while resilience is an extensive, multifaceted concept, it is "mainly defined by coping adaptively with traumatic stressors" (Schwarzer \& Warner, 2013, p. 140). While certainly not unrelated to resiliency, personal and collective efficacy beliefs provide an avenue to examine family strengths that may exist even in the absence of stressors or trauma (Bandura, 2000).

\section{Family Efficacy and Family Functioning}

Efficacy beliefs refer to an individual's or a group of individuals' beliefs in the ability to perform behaviors to produce desired outcomes (Bandura et al., 2011). The construct is derived from social cognitive theory, which frames human development and change as an agenetic process. Individuals and groups are agents who have the capacity to influence their functioning and experiences; efficacy is belief in their ability to do so. Efficacy beliefs are the "central mechanism" through which human agency is exercised (Bandura et al., 2011).

Within the family group, efficacy beliefs are interconnected indicators of family functioning (Caprara, Regalia, Scabini, Barbaranelli, \& Bandura, 2004). Bandura (2006) and Bandura et al. (2011) have suggested that individual efficacy beliefs, such as self-efficacy or parenting efficacy, contribute to a sense of collective family efficacy. In particular, parenting efficacy fosters healthy parental behaviors and plays an important role in child development. Parenting efficacy is also related to a lower level of family stress and reduced behavioral problems in children. Similarly, adolescent filial efficacy, which refers to a child's capability to establish and maintain good parental relationships while maintaining self-autonomy, is related to family satisfaction, open communication, better parental monitoring, and fewer escalated family conflicts (Caprara, Pastorelli, Regalia, Scabini, $\&$ Bandura, 2005).

The construct of family efficacy suggests that the family works collectively as an interconnected unit to overcome various situations and conflicts. Parenting efficacy, for example, is linked to positive parenting behaviors and constructive strategies during family crises (Leidy, Guerra, \& Toro, 2012). Jarrett (1997) noted that family and parenting efficacy buffered disadvantaged African American adolescents from risks associated with inner-city residency. In an environment with supportive parenting efficacy, children are more likely to complete high school, forgo premature childbearing, and participate in prosocial activities (Leidy et al., 2012). Furthermore, studies show that family efficacy is effective in raising children who practice healthy behaviors. For example, a close parentchild relationship is related to lower levels of marijuana use and risky sexual behaviors among children (Lac et al., 2011). Parental connectedness and involvement are recognized 
as effective parenting strategies for combatting risky behavior during adolescence (MogroWilson, 2008; Turner \& Sanders, 2006).

Overall, research suggests that family efficacy is an important construct that could be used effectively to promote adolescent health behaviors (Bandura et al., 2011; Kao et al., 2014; Profugo et al., 2009). But operationalization is limited by insufficient understanding of the genesis and role of family efficacy within diverse families. Few studies have examined family efficacy within diverse populations, and understanding of how efficacy is formed and framed within the context of cultural and familial values is limited.

\section{Family Functioning within the Familial and Cultural Context}

Research (Hwang, Wood, \& Fujimoto, 2010; Khafi, Yates, \& Luthar, 2014) has shown that patterns of family functioning differ due to families' varied cultural perspectives and values, and may affect familial interactions and behavior. For example, in a study of 167 Mexican-origin adolescent mothers and their own mothers, culturally rooted co-parenting profiles pertaining to communication, involvement, and conflict were closely related to the depressive symptoms and parenting efficacy of both daughters and their mothers 10 months after the adolescent mothers gave birth (Perez-Brena, Updegraff, UmañaTaylor, Jahromi, \& Guimond, 2015).

In particular, studies have consistently verified the influence of familial/cultural beliefs and values on adolescent health behaviors, including substance use and sexual behaviors (Kao, Loveland-Cherry, Guthrie, \& Caldwell, 2011; Sang, Cederbaum, \& Hurlburt, 2014). Conservative cultural beliefs emphasizing family loyalty, elder respect, and obedience may influence parent-child interaction patterns, particularly with regard to culturally sensitive issues such as intimate relationships, sexual expectancy, and psychological challenges (Calzada, 2010). The cultural beliefs of collectivism and individualism both play a significant role in family functioning and its interplay with adolescent health behaviors. In a study of 223 immigrant Hispanic adolescents, collective family functioning was protective against smoking behaviors (Lopez et al., 2010). Among Hispanic families living in the United States, degree of assimilation to Western cultural beliefs such as individualism was linked to parent-adolescent dynamics and indirectly influenced adolescents' substance abuse (Martinez, 2006). Both family structure and collectivism beliefs are also noted to play a significant role in parental support, communication, and monitoring, and have been found to subsequently influence Hispanic American adolescents' substance abuse (Wagner et al., 2010). Meanwhile, individualism, which stresses autonomy and relatedness, was also found to significantly influence family functioning (Kagitcibasi, 2005).

Socioeconomic status (SES) might also influence family functioning. Families of lower SES may engage different forms or patterns of family functioning to tackle financial and circumstantial challenges such as unjust treatment and societal/peer pressure (Aldridge, Shute, Ralphs, \& Medina, 2011; Jaffee et al., 2007). It is also possible that in the process of meeting familial needs, such families may develop certain strengths to address undue challenges.

In all, "optimal" family functioning may be defined very differently according to family culture, structure, and needs. Understanding sources and uses of collective efficacy and parent-adolescent dynamics among diverse families can shed light on how to design and engage strengths-based interventions to promote adolescent health behaviors.

\section{Current Study}

To summarize, families are interdependent systems that operate with beliefs in their members' abilities to work together to promote each other's well-being, development, and 
overcome difficulties. Efficacy beliefs and patterns can be influenced by the familial and cultural context. The purpose of this descriptive qualitative study was to examine and define sources of family efficacy within ethnically diverse families, evaluating how they develop and exercise family efficacy with the intent to promote health and inhibit risky behavior during adolescence.

The framework of symbolic interactionism provides an opportunity to understand parent-child interactions within the family system (Solomon, 1983). As with social cognitive theory, the framework describes individuals as active agents in relation to their environment or context. Within this framework, collective family efficacy beliefs center on family members' operative capabilities. Specifically, family members' interactive dynamics create an emergent property that is more than the sum of their individual attributes. It is posited that a family's structure and functioning contribute to its interactive family efficacy (Caprara et al., 2005). In this study, we examined family efficacy from the perspectives of African American, Chinese American, and Caucasian adolescents and their parents, evaluating how families engage efficacy to promote adolescents' health status and prevent risky behavior (i.e., early sexual activity; alcohol and marijuana use). To assess sources of family efficacy, we examined parent-adolescent interactions, how adolescents and parents define "family efficacy" within their family and cultural contexts, and how families engage strategies to handle difficulties such as personal or familial conflicts, unjust treatment, and undue societal or peer pressure. We then assessed how families engaged these sources of strength to promote health and prevent risky behavior.

\section{METHOD}

We used an ethnographic approach (Fetterman, 1989) to understand perceptions of family efficacy, cultural influences, and family dynamics and strategies. Ethnography dictates that research begin with an understanding of an individual's cultural perspective, and strives to avoid imposition of the external perspective of the researcher. In particular, our interview guide was developed by an ethnographic approach emphasizing the necessity of understanding individuals' cultural perspectives. Throughout the research process, we considered the emic and etic, or "insider" and "outsider," perspectives of culturally related interpretations.

\section{Participants and Recruitment Procedure}

Study procedures were reviewed and approved by the institutional review board of the sponsoring university. Using presentations and flyer distribution, we targeted two public middle schools in an ethnically diverse, lower income community where over $50 \%$ of students are African American and more than 60\% of students are eligible for free lunch (per family income). Because our goal was to assess family efficacy among three ethnically diverse groups, we opted to recruit Chinese American participants for our convenience sample, as they were an accessible subgroup of Asian Americans. Given prior investigations with this population (Kao \& Martyn, 2014; Kao \& Salerno, 2014), to successfully recruit Chinese American families, we targeted a local Chinese language center where the principal investigator has provided years of volunteer service. Eligible participants included adolescents aged 12-14 and one or more of their parents, all of whom had resided in the United States for at least 10 years. If eligible families had more than one child who fit the inclusion criteria, we asked the family to determine who would participate.

Before enrollment, prospective parents reviewed study information, involvement, and and their children's rights as study participants. All potential participants were informed that interview content would not be shared without permission, and that aliases would be 
used throughout the analysis and reporting process. If any member of the parent-adolescent dyad/triad had any unresolved issues with the study requirements, the family was not eligible to participate.

A total of 31 parent-adolescent dyads/triads participated in the study $(N=78)$. Table 1 presents a demographic summary. Ethnic breakdown of adolescent participants was: 11 Chinese American, 10 African American, 8 Caucasian, and 2 multiethnic (African American/Caucasian). The mean age was $12.68(S D=.748)$. Of the 28 mothers and 19 fathers, 3 were stepparents. Twenty-one adolescents reported living in a home with married or remarried parents, four adolescents lived with a divorced or separated parent, and six adolescents lived with single mothers who had never been married. Of these six single-parent families, three mothers (one who was Caucasian, African American, and multiethnic, respectively) had experienced homelessness previously. Half of the African American adolescents in our sample reported living in a single-parent or divorced/separated household. Families reported varied SES, as based

TABLE 1

Demographic Data

\begin{tabular}{|c|c|c|c|c|c|}
\hline & $\begin{array}{l}\text { Caucasian } \\
\text { American }\end{array}$ & $\begin{array}{c}\text { African } \\
\text { American }\end{array}$ & $\begin{array}{c}\text { Chinese } \\
\text { American }\end{array}$ & Multiethnic & $\begin{array}{l}\text { Total } \\
N(\%)\end{array}$ \\
\hline \multicolumn{6}{|c|}{ Age $(M=12.68, S D=.748)$} \\
\hline 12 years & 5 & 7 & 3 & 0 & $15(48.4)$ \\
\hline 13 years & 2 & 2 & 5 & 2 & $11(35.5)$ \\
\hline 14 years & 1 & 1 & 3 & 0 & $5(16.1)$ \\
\hline Subtotal & $8(25.8 \%)$ & $10(32.3 \%)$ & $11(35.5 \%)$ & $2(6.5 \%)$ & 31 \\
\hline \multicolumn{6}{|l|}{ Gender } \\
\hline Male & 4 & 4 & 5 & 1 & $14(45.2)$ \\
\hline Female & 4 & 6 & 6 & 1 & $17(54.8)$ \\
\hline Subtotal & $8(25.8 \%)$ & $10(32.3 \%)$ & $11(35.5 \%)$ & $2(6.5 \%)$ & 31 \\
\hline \multicolumn{6}{|l|}{ Parent marital status } \\
\hline Married/remarried & 6 & 4 & 10 & 1 & $21(67.7)$ \\
\hline Single & 2 & 3 & 0 & 1 & $6(19.3)$ \\
\hline Divorced/separated & 0 & 3 & 1 & 0 & $4(13)$ \\
\hline Subtotal & $8(25.8 \%)$ & $10(32.3 \%)$ & $11(35.5 \%)$ & $2(6.5 \%)$ & 31 \\
\hline \multicolumn{6}{|l|}{ Fathers' education level } \\
\hline$<12$ th grade & 0 & 2 & 0 & 0 & $2(10.5)$ \\
\hline High school & 1 & 0 & 0 & 0 & $1(5.3)$ \\
\hline Some college & 2 & 2 & 0 & 0 & $4(21)$ \\
\hline College & 1 & 0 & 1 & 0 & $2(10.5)$ \\
\hline Post college & 0 & 0 & 9 & 1 & $10(52.6)$ \\
\hline Subtotal & $4(21 \%)$ & $4(21 \%)$ & $10(52.6 \%)$ & $1(5.3 \%)$ & 19 \\
\hline \multicolumn{6}{|c|}{ Mothers' education level } \\
\hline$<12$ th grade & 0 & 3 & 1 & 1 & $5(17.9)$ \\
\hline High school & 0 & 0 & 1 & 0 & $1(3.6)$ \\
\hline Some college & 1 & 2 & 0 & 1 & $4(14.3)$ \\
\hline College & 2 & 4 & 3 & 0 & $9(32.1)$ \\
\hline Post college & 3 & 0 & 6 & 0 & $9(32.1)$ \\
\hline Subtotal & $6(21.4 \%)$ & $9(32.1 \%)$ & $11(39.3 \%)$ & $2(7.1 \%)$ & 28 \\
\hline \multicolumn{6}{|l|}{ Family income } \\
\hline$<\$ 29,000$ & 1 & 6 & 0 & 1 & $8(27.6)$ \\
\hline$\$ 30,000-\$ 49,999$ & 1 & 2 & 2 & 0 & $5(17.2)$ \\
\hline$\$ 50,000-\$ 99,999$ & 2 & 2 & 5 & 0 & $9(31)$ \\
\hline$>\$ 100,000$ & 2 & 0 & 4 & 1 & $7(24.1)$ \\
\hline Subtotal & $6(20.7 \%)^{\mathrm{a}}$ & $10(34.5 \%)$ & $11(37.9 \%)$ & $2(6.9 \%)$ & $29^{a}$ \\
\hline
\end{tabular}

Note. All of the Chinese American parents had emigrated from China or Taiwan.

a Two families (both Caucasian) chose not to report family income. 
on family income and parent education levels. Although the Chinese American families reported significantly higher SES than other families in the study, they brought unique acculturation experiences to the dataset. All the Chinese American parents were first-generation immigrants who had emigrated from China or Taiwan. Two African American parents had emigrated from South Africa and Dominica, respectively. All adolescents were born in the United States.

\section{Interview Procedure and Data Collection}

We conducted semi-structured interviews with adolescents and their parent/s simultaneously but separately in different rooms at the university site. Complimentary childcare was provided for families with other children. Interviews lasted approximately 1 hour. To ensure accuracy of data collection and analysis, trained interviewers took field notes to record all observations and nonverbal cues. Two digital recorders were used to record all interviews; audio files were transcribed and verified by two independent research assistants (RAs). Before analysis, the PI listened to all audio files and randomly audited at least $50 \%$ of the transcripts.

Interviews focused on learning about participants' perceived family strengths, family interactions, perceived health risks, and strategies for handling difficulty. An interview guide was used, and consisted of questions (detailed below) based on Bandura's concept of family efficacy. To ensure interviews were age-appropriate and culturally relevant, the guide was pretested and standardized with two mother-adolescent dyads (one African American and one Chinese American). We encouraged participants to freely describe their health concerns, potential health risks, and any personal or family difficulties and, to limit self-reported bias, asked them to share experiences and stories only to the degree that they felt comfortable.

Parents completed demographic sheets reporting age, gender, racial/ethnic background, and family income and structure. Adolescents completed event history calendars (EHC), rating their attitudes, behaviors, and perceived parental attitudes toward alcohol and marijuana use and sexual activity during the prior 2 years. We used a similar calendar in a prior study (Kao \& Martyn, 2014; Kao \& Salerno, 2014). During analysis, EHC data were coded, summarized, and compared with interview data.

\section{Interview 1}

We first asked participants to share a family story or an experience that demonstrated how they felt proud to be a part of their family. We then asked them to describe their family strengths, family values, and how they utilized such strengths (or values) to handle difficulties and to remain healthy, via questions such as "What makes your family strong?" and "What do you consider to be your family's strengths?" To assess parent-child interactions, we asked adolescents to share a story about how they interact with their parents and what they think about their parental relationship/s. We asked parents to share how they learn about their adolescents' whereabouts. Both parents and adolescents were asked to describe a typical day for their families, or to describe how their family spends time together. We also asked participants to talk about their family and cultural values that make them feel "proud to be part of your family (or culture)." We asked participants to discuss strategies they use to handle personal or family conflicts, or difficulty. Participants were instructed to define the term difficulty as they wished. Finally, we asked participants to associate their reported family strengths with their health perceptions and attitudes toward risky behaviors via questions such as "What do you do as a family to keep your family healthy?" and "What do you do to keep your adolescents from risky behaviors such as alcohol and marijuana use and early sexual activity?" 


\section{Interview 2}

The first interview focused on encouraging participants to share stories and experiences using open-ended, probing questions; the second interview (4-6 months later) was more structured. We focused on verifying and clarifying findings from the first interview as well as assessing changes in family events and dynamics. Before the second interview, we reviewed all transcripts and field notes from the first interviews and selected specific probing questions from the interview guide to address areas we wished to highlight. If participants identified any significant change in their family events or dynamics at the second interview, we asked them to explain the reason for such change and to elaborate on the way they felt the family had adjusted. We also asked participants to talk about how they used their family strengths to develop strategies to improve their health at home. Participants were told to self-define the term health.

The second round of interviews was conducted with 29 of the original participating families. Two families (one Chinese and one Caucasian) did not complete the second interview due to relocation. Data from all the interviews were included in our analysis. Participants received a $\$ 30$ cash incentive for participating in each interview. To maintain the privacy of participants, we used aliases throughout the analysis and reporting processes.

\section{Data Analysis}

Our study was informed by Bandura's construct of collective efficacy, which is rooted in social cognitive theory. We used thematic analysis to develop understanding of the meanings and sources of family efficacy. We used NVivo10 to conduct three rounds of coding, highlighting key themes within our qualitative data (Welsh, 2002). In the first round, three coders (the PI and two RAs) developed primary codes identifying sources of family strength. Coders independently read and coded transcripts, meeting multiple times to discuss and resolve discrepancies. New codes were created as necessary. Once coders achieved inter-rater agreement (Cohen's kappa $=.78$ ) (Smeeton, 1985), coders reread, categorized, and coded transcripts with consistent labels. Coders applied descriptive coding (Miles \& Huberman, 1994) and structural coding (MacQueen, McLellan-Lemal, \& Milstein, 2008) to label and index the concept of family efficacy. These codes were later clustered into the three domains of family efficacy that emerged from analysis.

Round 2 focused on understanding family dynamics, family functioning, and congruency among family members. Grouping the family as a unit, we coded, examined, sorted, and cross-examined responses from all family members using analytical memos (Birks, Chapman, \& Francis, 2008). We linked information obtained from the family demographic sheets, interview transcripts, field notes, and adolescent EHC data. Patterns of association between the three identified behaviors were very consistent-for example, adolescents more accepting of alcohol use were more accepting of early sexual activity. Therefore, we used their quantified EHC scores as representative "snapshots" of their overall risky behavior trends.

Major themes, annotations, and relational patterns that emerged from the analytic memos were used to finalize the three domains of family efficacy. We then used thematic analysis to pinpoint, examine, and record patterns describing how and where the families drew their family strengths (Braun \& Clarke, 2006). We used data triangulation to integrate findings from family members. To capture a more complete and holistic interpretation of family efficacy, we used participant descriptions of how and from where they drew strength when facing challenges.

Lastly, we used magnitude coding (Weston et al., 2001) to add a supplement to the three family efficacy domains. Two researchers reread all available materials from each participant and separately assigned a numerical value (range: $1=$ none to $4=h i g h$ ) to 
reflect the frequency and intensity of the family efficacy domains expressed by participants in the interviews. We had a good inter-rater correlation $(r=.72)$ (Landis \& Koch, 1977), so we used the mean score to indicate the magnitude of each family efficacy domain for each participant and family. We triangulated this value with adolescents' quantified $\mathrm{EHC}$ data, giving us a picture of the relationship between the family efficacy domains and adolescent risk perceptions and behavior. In all, we examined, compared, and integrated the categorization of these three domains of family efficacy to obtain nuanced understanding about how each family efficacy domain is utilized and how it relates to participants' cultural backgrounds, health risk perceptions, family dynamics, and family strategies.

\section{RESULTS}

Thematic analyses revealed three domains of family efficacy: relational, pragmatic, and value-laden. We categorized, named, and defined these domains according to their sources and characteristics. Overall, the sources of family efficacy emphasized and utilized by participants were very consistent between parent-adolescent dyads/triads. Below, we present narratives drawn from our qualitative interviews that define and illustrate the three domains of family efficacy described by our sample of diverse families. We then present findings pertinent to how these families utilized efficacy domains to inhibit adolescent risky behavior.

\section{Relational Family Efficacy}

Relational efficacy is the resilience generated from family relationships. Families who stressed the relational aspect of family efficacy tended to report satisfaction with communication. When dealing with conflict, they often used strategies such as "talking through problems" and "staying together." In all, parental connectedness, communication, cohesiveness, and parental support marked their descriptions of family efficacy, as detailed in the following narratives.

\section{Parental connectedness}

Alice (age 12, Caucasian) reported a close relationship with both parents, stating that this closeness helped her in many ways. When asked what made her family strong, she replied, "We like being involved in each other's lives." The family vacationed together and shared dinners where they "talk about our days." Alice reported having positive emotions when her mother asked her questions about "what I do throughout the day." Alice said the strong parental connection was the reason she listened to and obeyed her parents' rules about healthy eating, remaining active, and avoiding early sexual activity and substance use.

\section{Communication}

Alice's family also drew strength from open and effective communication. Her father also treasured their trusting relationship. "I want her to feel like I'm accessible," he said. "She can trust me, she can talk to me, and she can tell me things." He and Alice talked about and attended events together frequently. Alice's mother also emphasized the importance of having her feel comfortable talking with her about any topic. Alice noted that whenever members of her close-knit family faced conflicts or undue social/peer pressure, they "think of something positive and...talk to each other about the problem and how we feel."

Similarly, Rachel (age 12, Chinese American) listed her family's strengths as "close," "trusting," and that they "joke around" with each other. She described a close maternal 
relationship and wanted her mother to know everything because "I feel only safer with her knowing everything and she wants to know." Rachel's father also described a close relationship with his daughter, noting that they "talk about different things [such as] school and her friends."

\section{Cohesiveness}

Rachel's parents utilized their strong familial relationships to cope with the death of a grandmother who lived in Taiwan. Rachel recalled how the family's cohesiveness helped them deal with the death and to minimize the regret they felt because they lived far away. During the "memorable" trip to Taiwan for the funeral, Rachel felt close with her family. While a sad occasion, the family made use of the trip as a time to support each other. Rachel's mother was happy that her daughter used the opportunity to "get to know" her relatives. Their close relationships and connection with the extended family helped them deal with loss.

\section{Parental support}

John (age 13, Caucasian) shared how paternal support helped him deal with the dissolution of his parents' 25-year marriage. In the first interview, John expressed anger, frustration, and fear of losing his close connection with his father, who was no longer at home. He mostly directed his anger at his mother. She was "too overprotective" and he "did not understand her behavior" when she interacted with his father. In the second interview, however, John said that he was able to cope with his parents' divorce to a degree, mainly because of his father's support. Even though he was not living with his father, they shared interests and engaged in activities together. He felt his father's continuous support allowed him to cope with the stress of his parents' separation and divorce. Compared to his presence at the first interview, John appeared more relaxed and was able to share stories reflecting positive parent-child interactions.

\section{Pragmatic Family Efficacy}

Pragmatic efficacy is the competency generated by the way a family operates as a unit-what it does to operate effectively as a family, including rituals or routines such as spending time together, maintaining family boundaries, and parental monitoring. Many parents believed they were safeguarding their children's futures by exercising authority and implementing structure.

\section{Parental involvement and time together}

Joseph (age 13, Chinese American) identified parental involvement as a source of family strength, saying, "I could always count on them if I ever faced any problems." He described his parents as task-oriented; his mother oversaw his academics, his father his extracurricular activities. To maintain connection with his son, Joseph's father believed it was important to be involved in a shared interest. He often took time off work to drive Joseph to tennis practice and matches. He believed time together made the family strong:

I think we spend time with [the] kids and we take care of them most of the time and we understand what he is doing and what he-where he is so that gives him the safety feeling so he has a good healthy family to grow up.

Being present at these events was meaningful for Joseph. Although his parents did not regularly show physical signs of affection, Joseph thought their presence provided structure and security.

One immigrant family who struggled with social and cultural adaptation engaged the practice of being present to overcome difficulties. Lindsay (age 12, Chinese American) was 
diagnosed with Asperger syndrome around age 11. Limited understanding of her condition created many conflicts in the family. For the mother, the diagnosis brought sorrow and feelings of inadequacy. To compensate, she became very involved with her daughter's daily life. She admitted that she was "often too involved," particularly with Lindsay's homework.

Lindsay's father, meanwhile, grew up in a Chinese culture in which the father is expected to play a firm and disciplinary role in the family. A year before the interview, he got frustrated and lost his temper when he could not help his daughter understand "a simple math problem." The school accused him of physically abusing Lindsay, and social workers were appointed to her case. The mother said the ordeal almost "destroyed my family." They felt "misunderstood" by the school and by society in general. Recalling the experience, Lindsay defended her family structure, saying, "They had no right to intrude [on] my family like that." To cope, the family drew strength from quality time spent together. Although the father had difficulty expressing his feelings, he was involved with Lindsay's favorite extracurricular activity. Lindsay remarked, "I know my father cares about me because he is always picking me up after the club. [My parents] help me with my homework." Forms of pragmatic efficacy helped the family handle the stress of being an immigrant family and having a child with special needs. During the second interview, the mother reported that they had taken several family trips and felt closer to each other. Both Lindsay and her mother shared that they were talking more about their days with each other.

\section{Setting boundaries and parental monitoring}

The father of twins Diane and Vivian (age 12, African American) set rules for his girls, such as not being allowed to make personal calls on their cell phones and only visiting homes of friends whose parents he knew. The father had emigrated from Dominica 8 years before the girls were born; he commented, "Back home, the community can raise your kids, but not in here...thus [parents] have to be extra careful here." The twins did not report problems following parental rules. Diane described her family's strengths as "trusting each other, spending time together, and never giving up." Vivian said her parents were very involved with their schooling; they helped with homework and made decisions about school-related activities. Regular family activities and consistent rules provided stability and strength, including having the girls help make dinner and going to the movies each week.

Similarly, Susie's (age 12, Caucasian) family established family efficacy via firm parenting practices. Despite hectic schedules, they ate together every Sunday; the family rule was that no friends could come over on that day. Susie's father explained their "family council":

We sit down with the calendar to go over the next week and figure out where everyone has to be and how they're going to get there....It's also an opportunity for any interesting issues...it usually winds up being pretty fun and pretty rowdy. But this is also an opportunity to talk about. . .our relationship and about things...that happen in our family.

Susie thought this practice was "very meaningful" and allowed her to connect with her family members. She also said that going to church together helped them draw strength from religion and faith in God. When facing undue societal/peer pressure and/or unjust treatment, Susie said the family would "pray and read our Scriptures"-an element of value-laden family efficacy.

\section{Value-Laden Family Efficacy}

Value-laden efficacy refers to effectiveness generated from values family members honor. Families may derive values from cultural or religious beliefs. Examples reported by 
participants include practicing a religious faith, upholding family/ethnic pride, and working hard.

\section{Endorsing values and religious faith}

Devon (age 12, African American) lived with his mother and stepfather. The family overcame many obstacles, including homelessness and a grandmother's struggle with substance abuse. The family drew strength from the values of devotion, determination, and faith in God. Although Devon did not like "the history of drugs in his family," he was determined "to change it." He commented that his family drew strength from God. He shared that his family "goes to church almost every week" and he said he was working to not "mess up the relationship between me and God because I don't want to go to Hell."

The family demonstrated other elements of value-laden efficacy. The mother discussed her experience with homelessness and shared how she drew strength from her value system:

I think my [godmother]... taught me a lot of lessons growing up about love, obligation, responsibility, about not always seeing things happen, knowing when to be places and know when to not be there and be honest, I try to pass things like that to my own kids.

Devon commented that in his family "we all got each other's back." He recalled that his mother taught him to "keep pushing for your goal - that you want to do, never give up." Similarly, his stepfather told him to "be a hardworking man....Go to work, don't sell drugs. Keep your mind clean, keep everything clean." These values helped the family overcome many family conflicts.

\section{Collective attitudes, ethnic pride}

Frank's (age 14, African American) family endorsed a collective attitude. His mother recalled that they had uprooted the family and moved to the city to save her mother's home. It was a challenging experience, but they had to help. Frank felt this grandmother was a source of the family's collective strength: "she keeps everyone together." He believed his culture led him to value being "strong" and "a leader for others." Frank's father told him about how his "family's unique spelling of our last name" made their family "strong and independent"; it made him "proud to be a part of my family and my culture." The family also drew strength from religious values. Frank's mother shared: "We mainly tackle our challenges through prayer and then proceed accordingly" and "Families that pray together stay together." Overall, values of collectivism and prayer strengthened the family and nourished family efficacy.

A similar sense of ethnic pride was also observed in many Chinese American families. They spoke about the meaning of being an Asian living in the United States and how they strived to live up to high but sometimes unrealistic expectations of Asian Americans.

\section{Working hard}

Many families endorsed values of working hard and having high expectations. Steve (age 13, Caucasian), for example, respected and followed his stepfather's values of "hard work and [being] in control." They taught him "to be hard-working and not a deadbeat." Steve endorsed pragmatic and value-laden efficacy, stating, "Love alone is not going to teach your child what he needs to know through life....[Parents need to] be firm." He also validated his parents' religious involvement. Returning to church after a prolonged absence "changed" his life and gave him feelings of "self-discovery."

Steve's stepfather considered his faith to be the most important value. When facing family conflict, the stepfather said, "[We] look at the brighter side of things. We pray and sit down to talk to find a solution." Steve shared that his grandfather, with whom he was 
very close, suffered from Alzheimer's and later died of cancer, and that his family helped him through this by praying together, talking about death, and going out together. Steve reported that his family did many things together, and that seemed to ease the pain. These anecdotes demonstrate how this family drew efficacy from its religious faith and participation in collective activities.

\section{Utilizing Family Efficacy to Inhibit Adolescent Risky Behaviors}

Magnitude coding of patterns of family efficacy among the three family groups revealed that while all families exerted some pattern of family efficacy, individual families drew upon difference sources in different combinations to protect adolescents from risk. We observed variations based on cultural influences and background, prior experiences, and level of acculturation, defined as the process of cultural and psychological change that results when two cultures meet (Sam \& Berry, 2010). Notably, although families drew upon different efficacy sources in different combinations, many sought to engage and enhance multiple domains.

\section{Cultural influences and variations}

When asked to identify their family's cultural strengths, African American and Chinese American participants were usually quick to identify and relate their family efficacy to cultural beliefs/values. Caucasian participants, meanwhile, tended to require more time to think about or more clarification of the term culture. Ethnic minority families were more likely than Caucasian families to exert or endorse family efficacy that was rooted in their culture and their ethnicity. Heavily endorsed values included collectivism; "familyfirst"; Black or Asian "pride"; and respecting elders.

When asked what they did to protect their adolescents from risk, many Caucasian participants were quick to mention the importance of maintaining an open and trusting relationship between parents and children. Caucasian parents appeared to favor relational efficacy elements, and often drew strength from this domain to prevent their children from risky behaviors. Ethnic minority parents in the study, meanwhile, tended to rely on the pragmatic domain of efficacy to protect their children from risky situations. Many Chinese American parents, for example, used parental involvement, regular routines/rituals, firm family boundaries, and shared family activities to promote their children's health behaviors, while many African American parents used strict parenting practices, firm rules/ discipline, and close parental monitoring to protect their children from risky situations and behaviors.

Regardless of ethnic/cultural background, many families consistently tapped into faith and religious beliefs or values. Although religious beliefs or faiths varied, a majority of families engaged value-laden efficacy via prayer, going to church/temple, or reading religious texts.

\section{Experience influences implementation}

Past experiences played an important role in how and what domains of efficacy parents engaged to protect their adolescents from risky health behaviors. Compositions of family efficacy varied according to a family's cultural background and past experiences, including incarceration, homelessness, teen pregnancy, substance abuse. Parents who had themselves engaged in risky behavior often tapped into more than one domain of family efficacy and in differing sequences to prevent their children from such behavior.

Many Caucasian parents emphasized parent-child communication, reporting that they spoke with their adolescents about personal experiences with dating or substance use, such as strategies they employed or mistakes they themselves had made. Caucasian 
parents engaged relational efficacy in an attempt to "persuade" their children from risky behavior. Conversely, ethnically diverse parents who had experienced financial hardship were the least likely to endorse relational efficacy as a priority and/or rely on it solely. Instead, they tended to tap into the pragmatic and value-laden domains. To protect children from risk, specifically unprotected sex and substance use, many African and Chinese American parents promptly and frequently mentioned the use of strategies relevant to pragmatic efficacy, such as parental monitoring. Minority parents of lower SES, particularly single parents, said they used value-laden efficacy to help their children stay strong and avoid temptation and deviant peers.

It was also evident that utilization of family efficacy domains was affected by families' or family members' degree of acculturation. Some less acculturated Chinese American parents, for example, were concerned that they did not sufficiently express their feelings. Joseph's mother expressed a "lack of confidence" as a mother living in a culture that places heavy emphasis on expressing parental warmth. She grew up in a culture where parents' primary responsibility is to "teach and prepare" their children so they will have a brighter future. She reflected that she was "strict" and not as "warm" as other parents. Her ineptness in relational efficacy sometimes made her feel she is "not a good enough mother."

\section{Families strive to enhance other domains of efficacy}

To improve adolescents' health behaviors, many families utilized one efficacy domain to enhance another. For example, many parents used relational efficacy to first establish a strong parent-child relationship and then drew upon pragmatic efficacy to establish rules to keep their children safe. Parents who placed a high priority on religious or cultural beliefs hoped that their value-laden efficacy would enrich elements of pragmatic efficacy and thereby protect their adolescents from risky behaviors. The main goal for these parents was to transmit their value-laden efficacy to the next generation.

Families who demonstrated multidimensional efficacies-those who were skilled at implementing all three domains - were more likely to report healthier behaviors and to have coping strategies in place to deal with family difficulties. When a family lacked a particular domain of family efficacy, however, family members were likely to notice this deficit. For example, Vincent's (age 13, Chinese American) parents employed strict parenting practices and were not very expressive in their relationships with their children. His mother described herself as a "tiger mom" and felt that strict parenting was necessary to prepare children for the future. Vincent, meanwhile, had a negative perception of his parental relationships, stating, "I can only think of the bad things, I can't really think of the good things." He wished he could be like his "white peers," who have "fun parents" who "cut them slack from time to time." We consistently observed this theme in adolescents who longed for increased relational family efficacy.

Similarly, adolescents in families less skilled in pragmatic family efficacy tended to crave more parental involvement and/or monitoring, such as rules and rituals that could provide greater stability within the family. Bob (age 13, African American) lived with his mother and wished she was more involved in his school activities. "I wish my mom is not always working," he said, and wished she could "spend more time" helping with his homework so he could get "better grades."

\section{DISCUSSION}

This study evaluated how a sample of ethnically and socioeconomically diverse families develop and exercise efficacy with the intent to protect adolescents from risky health behaviors. Three rounds of coding revealed that families engaged elements of one or more domains of family efficacy: relational, pragmatic, and value-laden. This process allowed us 
to examine sources of family strength from the perspective of diverse adolescents and their parents. The main similarity among the three ethnic groups was parents' efforts to utilize the aspects of family efficacy with which they were most familiar and comfortable. The differences, however, were rooted in both cultural, religious, and familial beliefs and parents' prior experiences as children and as parents. For example, parents often used beliefs such as individualism or collectivism to illustrate their perspectives about family efficacy. Their cultural, religious, and/or familial beliefs were often used to determine family rules or boundaries about what was or was not allowed.

Even though not all families were skilled at drawing strength from the relational efficacy domain, this was the domain that was mentioned most frequently by all families. We had anticipated this finding, given widespread emphasis on the parent-child relationship in the United States. The societal expectation that parents should socialize children to learn, develop, and become mature individuals able to function in a complex society may influence parenting practices. Many Caucasian parents reported the necessity of helping their children develop skills to relate to and socialize with others, particularly the ability to display warmth or affection. This may explain why many Caucasian participants attributed familial satisfaction and dissatisfaction to the relational domain. Meanwhile, ethnic minority families, and those of lower SES in particular, were more likely to emphasize the pragmatic or value-laden domains to protect children from risky behavior and to help them develop resilience and overcome circumstantial challenges such as financial hardship, unjust treatment, and undue peer or social pressure.

Such variation in emphasis between ethnic groups may be the result of the challenges that minorities face that are embedded within the context of ethnicity, SES, and environment (Kotchick \& Forehand, 2002). It could explain why authoritarian parenting - that is, parenting marked by a high degree of authority and low degree of responsiveness to a child-has been found to be more protective for minority families (Kotchick \& Forehand; Pong, Hao, \& Gardner, 2005). It is also possible that variation in families' utilization of efficacy may be reinforced by parents' community experiences. Parents may compare their utilization of family efficacy to other members in the community including, for immigrants, families in or from their home countries. This constant comparison and reflection may influence parents' implementation of family efficacy to protect children from risk (Kao et al., 2014; Sang et al., 2014).

For ethnic minority and low-SES families in our study, environment, acculturation, and economic hardship presented unique challenges. We found that when facing pronounced difficulties, including financial hardship or undue social pressure or treatment, many minority families, particularly those of lower SES, drew strength from religious and/or cultural values. Families' exertion of value-laden efficacy mirror the resiliency described by Walsh (2003), in which disadvantaged families utilized their cultural and religious values to foster resilience. Our findings echo Walsh's notion that resilience is a culturally and contextually specific construct. Culturally embedded understanding of resilience is important for health professionals who work with marginalized and at-risk adolescents, as their resilience is highly influenced by environment and positive interactions (Ungar, 2008). For participants in our study, resilience was cultivated by a sense of familial belonging, perceived collective attitudes and ethnic pride established via parentchild interactions, and achieving personal meaning via cultural or religious belief. By recognizing the value-laden aspect of efficacy and maximizing families' culturally embedded resilience, family practitioners can help ethnically and economically diverse families tap into efficacies rooted in their familial, cultural, and religious beliefs and values. Future studies should consider a simultaneous or linked assessment of the concepts of resilience and family efficacy. 
Finally, we found many families drew strength from the domain/s with which they were most comfortable. Even though the composition of family efficacy may look different within different families, and the definition of "optimal" parenting may vary across cultural contexts, positive influences on adolescent health behaviors are evident. Understanding the different compositions and combinations of efficacy can allow family practitioners to incorporate family efficacy in health promotion strategies to maximize adolescent health behaviors.

\section{Limitations}

Our findings are drawn from a small convenience sample, and we are not able to capture all instances relevant to family efficacy. Generalizability is limited. Additional investigations about family efficacy are warranted, particularly with socioeconomically and ethnically diverse groups. Because we drew from different sample pools to successfully recruit participants, our sample was stratified along socioeconomic lines, Chinese American families recruited via a private language center reported generally higher SES than the African American and Caucasian families, who were recruited via local public schools. Further, different ethnic groups may face varied acculturation challenges and family conflicts (Kao et al., 2011). Direct comparison among groups is not ideal. Lastly, while our process of assessing the magnitude of each efficacy source within the family unit allowed us to assess how diverse families utilized different domains of family efficacy, potential bias may exist. Although we had good inter-rater reliability, future studies will need to refine the quantification of these three efficacy domains.

\section{Implications}

Our study contributes to the body of knowledge regarding the concept of family efficacy and its ability to affect adolescent health behaviors. Specifically, family efficacy provides an opportunity to identify, examine, and utilize sources of familial strength within families' cultural context. This knowledge enables practitioners to better understand and assess sources of familial strength that are rooted in diverse clients' family culture and background. Such an approach can help practitioners avoid bias, tap into existing family efficacy, and empower clients to maximize efficacy beliefs for healthy behaviors. In particular, practitioners can use this knowledge to broaden the scope of family assessments and design interventions suitable to diverse clients' cultural backgrounds and past experiences.

Categorizing family efficacy into three domains allows practitioners to focus on sources of strength without defining different or unfamiliar parenting practices and family functioning as "dysfunctional" or "less ideal." "Blame-the-parents" labeling (Aldridge et al., 2011) discourages and disempowers. A strengths-based approach, which assumes that familial strength is generated from self-realization of a family's own strengths (Kuokkanen \& Leino-Kilpi, 2000), might be more appropriate for ethnic/racial minority families and families of lower SES, who may face unique environmental, economic, and/or acculturation challenges (e.g., undue societal pressure and treatment). Although we are unable to establish a definite relationship between family efficacy and specific health behaviors, it is evident that ethnically diverse families and families of lower SES tap into varied domains of efficacy to ensure their children are safe and able to handle personal and family conflicts. It appears that, regardless of domain, families with higher efficacy are more likely to have strategies in place to promote adolescents' health behaviors and prevent risky situations, such as delinquent peer association. This finding is congruent with studies suggesting less structured parenting practices are associated with risky sexual behavior (Kao \& Manczak, 2013) and substance abuse (Mogro-Wilson, 2008) among adolescents. 
To operationalize the construct of family efficacy, more studies are needed to understand the value-laden aspect of family efficacy. Collective efficacy measures developed by Bandura and others (Caprara et al., 2005) have not focused on this domain. However, many ethnic minority families and families of low SES in our study reported its significance in their parenting practices, particularly when facing financial hardship or family conflicts. Further assessment of value-laden efficacy among minority families and those of lower SES is critical, especially for interventions that seek to engage value-laden efficacy to prevent adolescent risky behavior.

Family efficacy plays an important role in parents' efforts and strategies to ensure the health of their families. The domain/s from which families draw their efficacy is determined by cultural background and family beliefs and experiences. Future studies can extend this research by verifying the relationship between family efficacy and specifically identified health behaviors. Identifying and understanding these associations can help providers develop family-based interventions to effectively promote adolescent health behaviors.

\section{REFERENCES}

Aldridge, J., Shute, J., Ralphs, R., \& Medina, J. (2011). Blame the parents? Challenges for parent-focused programmes for families of gang-involved young people. Children \& Society, 25(5), 371-381.

Bandura, A. (2000). Exercise of human agency through collective efficacy. Current Directions in Psychological Science, 9, 75-78.

Bandura, A. (2006). Toward a psychology of human agency. Perspectives on Psychological Science, 1(2), 164-180.

Bandura, A., Caprara, G. V., Barbaranelli, C., Regalia, C., \& Scabini, E. (2011). Impact of family efficacy beliefs on quality of family functioning and satisfaction with family life. Applied Psychology, 60(3), 421448.

Birks, M., Chapman, Y., \& Francis, K. (2008). Memoing in qualitative research: Probing data and processes. Journal of Research in Nursing, 13(1), 68-75.

Braun, V., \& Clarke, V. (2006). Using thematic analysis in psychology. Qualitative Research in Psychology, 3(2), $77-101$.

Calzada, E. J. (2010). Bringing culture into parent training with Latinos. Cognitive and Behavioral Practice, 17(2), 167-175.

Caprara, G. V., Pastorelli, C., Regalia, C., Scabini, E., \& Bandura, A. (2005). Impact of adolescents' filial self-efficacy on quality of family functioning and satisfaction. Journal of Research on Adolescence, 15(1), 71-97.

Caprara, G. V., Regalia, C., Scabini, E., Barbaranelli, C., \& Bandura, A. (2004). Assessment of filial, parental, marital, and collective family efficacy beliefs. European Journal of Psychological Assessment, 20(4), $247-261$.

Fang, L., \& Schinke, S. P. (2012). Two-year outcomes of a randomized, family-based substance use prevention trial for Asian American adolescent girls. Psychology of Addictive Behaviors, 27(3), 788-798. doi:10.1037/ a0030925

Fetterman, D. M. (1989). Ethnography: Step by step, Vol. 17. Newbury Park, CA: Sage.

Hodge, D. R. (2001). Spiritual assessment: A review of major qualitative methods and a new framework for assessing spirituality. Social Work, 46(3), 203-214.

Hwang, W.-C., Wood, J. J., \& Fujimoto, K. (2010). Acculturative family distancing (AFD) and depression in Chinese American families. Journal of Consulting and Clinical Psychology, 78(5), 655-667.

Jaffee, S. R., Caspi, A., Moffitt, T. E., Polo-Tomás, M., \& Taylor, A. (2007). Individual, family, and neighborhood factors distinguish resilient from non-resilient maltreated children: A cumulative stressors model. Child Abuse \& Neglect, 31(3), 231-253.

Jarrett, R. L. (1997). African American family and parenting strategies in impoverished neighborhoods. Qualitative Sociology, 20(2), 275-288.

Kagitcibasi, C. (2005). Autonomy and relatedness in cultural context: Implications for self and family. Journal of Cross-Cultural Psychology, 36(4), 403-422.

Kao, T.-S. A., Loveland-Cherry, C., Guthrie, B., \& Caldwell, C. H. (2011). Acculturation influences on AAPI adolescent-mother interactions and adolescents' sexual initiation. Western Journal of Nursing Research, 33(5), $712-733$.

Kao, T.-S. A., Lupia, C. M., \& Clement-Stone, S. (2014). Family efficacy as a protective factor against immigrant adolescent risky behavior: A literature review. Journal of Holistic Nursing, 32(3), 202-216. 
Kao, T.-S. A., \& Manczak, M. (2013). Family influences on adolescents' birth control and condom use, likelihood of sexually transmitted infections. The Journal of School Nursing, 29(1), 61-70.

Kao, T.-S. A., \& Martyn, K. K. (2014). Compare Asian American and Caucasian American adolescents' perceived parental expectancies and their sexual behaviors. Sage Open, 4(2), 1-16. doi: 10.1177/2158244014535411

Kao, T.-S. A., \& Salerno, J. (2014). Keeping adolescents busy with extracurricular activities. The Journal of School Nursing, 30(1), 57-67.

Khafi, T. Y., Yates, T. M., \& Luthar, S. S. (2014). Ethnic differences in the developmental significance of parentification. Family Process, 53(2), 267-287.

Kotchick, B. A., \& Forehand, R. (2002). Putting parenting in perspective: A discussion of the contextual factors that shape parenting practices. Journal of Child and Family Studies, 11(3), 255-269.

Kumpfer, K. L., \& Alvarado, R. (2003). Family-strengthening approaches for the prevention of youth problem behaviors. American Psychology, 58(6-7), 457-465.

Kuokkanen, L., \& Leino-Kilpi, H. (2000). Power and empowerment in nursing: Three theoretical approaches. Journal of Advanced Nursing, 31(1), 235-241.

Lac, A., Unger, J., Basáñez, T., Ritt-Olson, A., Soto, D., \& Baezconde-Garbanati, L. (2011). Marijuana use among Latino adolescents: Gender differences in protective familial factors. Substance Use \& Misuse, 46(5), $644-655$.

Landis, J. R., \& Koch, G. G. (1977). The measurement of observer agreement for categorical data. Biometrics, 33(1), 159-174.

Leidy, M. S., Guerra, N. G., \& Toro, R. I. (2012). Positive parenting, family cohesion, and child social competence among immigrant Latino families. Journal of Latina /o Psychology, 1(S), 3-13.

Lopez, B., Huang, S., Wang, W., Prado, G., Brown, C., Zeng, G. et al. (2010). Intra-personal and ecodevelopmental factors associated with smoking in Hispanic adolescents. Journal of Child and Family Studies, 19(4), 492503.

MacQueen, K. M., McLellan-Lemal, K. B., \& Milstein, B. (2008). Handbook for team-based qualitative research. Plymouth, UK: AltaMira Press.

Martinez, C. R. (2006). Effects of differential family acculturation on Latino adolescent substance use. Family Relations, 55(3), 306-317.

Miles, M., \& Huberman, A. (1994). Qualitative data analysis. Thousand Oaks, CA: Sage.

Mogro-Wilson, C. (2008). The influence of parental warmth and control on Latino adolescent alcohol use. Hispanic Journal of Behavioral Sciences, 30(1), 89-105.

Perez-Brena, N. J., Updegraff, K. A., Umaña-Taylor, A. J., Jahromi, L., \& Guimond, A. (2015). Coparenting profiles in the context of Mexican-origin teen pregnancy: Links to mother-daughter relationship quality and adjustment. Family Process. 54(2), 263-279. doi:10.1111/famp.12115

Pong, S., Hao, L., \& Gardner, E. (2005). The roles of parenting styles and social capital in the school performance of immigrant Asian and Hispanic adolescents. Social Science Quarterly, 86(4), 928-950.

Profugo, D., Mendoza, S., \& Magno, C. (2009). Developing Asian values, self-construal, and resiliency through family efficacy and parental closeness. The International Journal of Research and Review, 1, 1-20.

Sam, D. L., \& Berry, J. W. (2010). Acculturation: When individuals and groups of different cultural backgrounds meet. Perspectives on Psychological Science, 5(4), 472-481.

Sang, J., Cederbaum, J. A., \& Hurlburt, M. S. (2014). Parentification, substance use, and sex among adolescent daughters from ethnic minority families: The moderating role of monitoring. Family Process, 53(2), $252-266$.

Schwarzer, R., \& Warner, L. M. (2013). Perceived self-efficacy and its relationship to resilience. In S. PrinceEmbury \& D. H. Soklofske (Eds.), Resilience in children, adolescents, and adults: Translating research into practice (pp. 139-150). Springer Science+Business Media: New York. doi:10.1007/978-1-4614-4939-3_10

Smeeton, N. (1985). Early history of the Kappa statistic. Biometrics, 41(3), 795.

Solomon, M. R. (1983). The role of products as social stimuli: A symbolic interactionism perspective. Journal of Consumer Research, 10(3), 319-329.

Turner, K., \& Sanders, M. R. (2006). Dissemination of evidence-based parenting and family support strategies: Learning from the Triple P-Positive Parenting Program system approach. Agression and Violent Behavior, 11, 176-193.

Ungar, M. (2008). Resilience across cultures. British Journal of Social Work, 38(2), 218-235.

Wagner, K. D., Ritt-Olson, A., Chou, C.-P., Pokhrel, P., Duan, L., Baezconde-Garbanati, L. et al. (2010). Associations between family structure, family functioning, and substance use among Hispanic/Latino adolescents. Psychology of Addictive Behaviors, 24(1), 98-108.

Walsh, F. (2003). Family resilience: A framework for clinical practice. Family Process, 42(1), 1-18.

Welsh, E. (2002). Dealing with data: Using NVivo in the qualitative data analysis process. Forum: Qualitative Social Research, 3(2), art. 26.

Weston, C., Gandell, T., Beauchamp, J., McAlpine, L., Wiseman, C., \& Beauchamp, C. (2001). Analyzing interview data: The development and evolution of a coding system. Qualitative Sociology, 24(3), 381-400. 